\title{
In vitro Effect of the Combination of Aztreonam and Amoxicillin/Clavulanic Acid Against Carbapenem-Resistant Gram-Negative Organisms Producing Metallo- $\beta$-Lactamase
}

This article was published in the following Dove Press journal: Infection and Drug Resistance

\author{
Qian $X u^{1,2, *}$ \\ Ying $\mathrm{Fu}^{3, *}$ \\ Jingshu $\mathrm{ji}^{3}$ \\ Xiaoxing $D u^{1,2}$ \\ Yunsong $\mathrm{Yu}^{\mathrm{I}, 2}$ \\ 'Department of Infectious Diseases, Sir \\ Run Run Shaw Hospital, School of \\ Medicine, Zhejiang University, Hangzhou, \\ Zhejiang Province, 310016, People's \\ Republic of China; ${ }^{2}$ Key Laboratory of \\ Microbial Technology and Bioinformatics \\ of Zhejiang Province, Hangzhou, Zhejiang \\ Province, 310016, People's Republic of \\ China; ${ }^{3}$ Department of Clinical \\ Laboratory, Sir Run Run Shaw Hospital, \\ School of Medicine, Zhejiang University, \\ Hangzhou, Zhejiang Province, 3100I6, \\ People's Republic of China
}

*These authors contributed equally to this work
Correspondence: Yunsong Yu

Qingchun East Road No. 3, Hangzhou

City, Zhejiang Province, 310016, People's

Republic of China

$\mathrm{Tel} / \mathrm{Fax}+86-57 \mathrm{I}-86006 / 42$

Email yvysI19@zju.edu.cn
Introduction: Antibiotics for treating infectious diseases caused by carbapenem-resistant Gram-negative pathogens (CR-GNOs) are very limited in clinical practice. We aim to provide supportive evidence by revealing the combined effect of aztreonam (ATM) and amoxicillin/clavulanic acid (AMC) against GNOs with carbapenem resistance mediated by metallo- $\beta$-lactamase (MBL).

Methods: All isolates were identified by the VITEK system and EDTA inhibitory assays. PCR followed by sequencing was conducted to confirm the genotypes of MBL and extended spectrum $\beta$-lactamase (ESBL). Time kill assay was performed to clarify the bactericidal effect of drug combination.

Results: A total of 59 MBL-producing CR-GNOs (33 Enterobacteriaceae spp. isolates and 26 Pseudomonadales isolates) were identified and there found three MBL genes, namely, $b l a_{\mathrm{IMP}}$, $b l a_{\mathrm{NDM}}$ and $b l a_{\mathrm{VIM}}$, with ratios of $76.2 \%, 11.8 \%$ and $11.8 \%$, respectively. The Enterobacteriaceae spp. isolates were commonly positive for the ESBL genes, including $b l a_{\mathrm{TEM}}$ (18 isolates), $b l a_{\mathrm{SHV}}$ (20 isolates) and $b l a_{\mathrm{CTX}-\mathrm{M}-1}$ ( 8 isolates), while the $P$. aeruginosa isolates were positive for $b l a_{\text {OXA-10 }}$ (11 isolates). The checkerboard microdilution assay was used to detect combination effect of ATM and AMC, which showed synergy (97.0\%) and partial synergy (3.0\%) in Enterobacteriaceae spp. isolates, and partial synergy (42.3\%) and indifference $(34.6 \%)$ in the Pseudomonadales isolates. Four Enterobacteriaceae spp. isolates were selected for a time-kill assay, and rapid bactericidal effects were observed in the combination groups compared to the control and mono-ATM groups; these effects began in the first hour and continued to the sixth hour, yielding a 5- to 7-fold reduction in $\log 10 \mathrm{CFU} / \mathrm{mL}$.

Discussion: The combination of ATM and AMC would be an available option to control infections caused by MBL-producing CR-GNOs, especially Enterobacteriaceae spp. isolates that coproduce ESBLs, and exhibit significant synergic effects in vitro.

Keywords: gram-negative pathogens, carbapenem resistance, metallo- $\beta$-lactamase, extended spectrum $\beta$-lactamase, aztreonam, amoxicillin/clavulanic acid, combination

\section{Introduction}

The production of metallo- $\beta$-lactamase (MBL) is an important mechanism of carbapenem resistance in Gram-negative pathogens (GNOs). ${ }^{1}$ It has placed significant burden on healthcare system since infections caused by MBL-producing GNOs (MBL-GNOs) have distributed globally during the last two decades and are always associated with increased 
morbidity and mortality. ${ }^{2}$ The human public health is seriously threatened due to very few effective antibiotics that were available for clinical treatment. ${ }^{3}$

Although MBL is capable of inhibiting a broad range of $\beta$ lactams, including penicillin, cephalosporins and carbapenems, it lacks the ability to hydrolyse monobactams, such as aztreonam (ATM). ${ }^{4}$ Therefore, antibiotic treatments based on ATM are considered a useful option for controlling GNOs that produce MBL. ${ }^{5}$ However, it is not suggested that ATM be used alone in clinical practice because of the complexity of resistance mechanisms in CR-GNOs. Increasing evidence has shown that resistance mechanisms, such as extended spectrum $\beta$-lactamases (ESBLs) and efflux pumps, that would directly lead to the failure of ATM widely exist in clinical CR-GNOs. ${ }^{6}$ Recently, ATM combined with $\beta$-lactam/ $\beta$-lactamase inhibitors (BL/BLIs) is becoming a prospective choice for treatment. ${ }^{7}$

The combination of ATM and BLIs or BL/BLIs has generated more interest in the medical community; among these inhibitors, ceftazidime/avibactam and amoxicillin/clavulanic acid (AMC) are the most promising options. ${ }^{6,8}$ It was reported that ATM combined with ceftazidime/avibactam or AMC could be an attractive but unconventional treatment for infections with MBL-producing Enterobacteriaceae. ${ }^{89}$ Nevertheless, compared to ceftazidime/avibactam, AMC is less expensive, which contributes to the relief of the economic burden. ${ }^{10,11}$ To provide more evidence for clinical treatment in our country, we study multiple species of CR-GNOs that produce MBL that were isolated from different hospitals and evaluate the combined effect of ATM and AMC in vitro by using the checkerboard microdilution method and time kill assays.

\section{Materials and Methods}

\section{Species Identification and Antimicrobial Susceptibility Test}

Carbapenem-resistant Gram-negative bacteria were collected from several tertiary hospitals in China between 2011 and 2016. The VITEK system (bioMérieux, France) was used for species identification and antimicrobial susceptibility testing (AST). The minimal inhibitory concentrations (MICs) of meropenem, aztreonam (Sigma Aldrich, USA) and amoxicillin/ clavulanic acid (4:1, Sigma Aldrich, USA) were determined with the broth microdilution method according to the standard procedure of the Clinical \& Laboratory Standards Institute (CLSI) guideline. ${ }^{12}$ Since the ratio of amoxicillin and clavulanic acid in our study was different from that recommended by the CLSI, the MIC of AMC was recalculated according to the 4:1 ratio, and the MICs of both amoxicillin and clavulanic acid were obtained. The breakpoint of amoxicillin in AMC in the CLSI was used to explain the result of AMC by using the MIC of amoxicillin recalculated as objectives. Considering no interpretation breakpoint of $P$. aeruginosa to AMC and of A. baumannii to ATM and AMC in the CLSI guideline, the breakpoint of $P$. aeruginosa to ATM was used to explain the AST of A. baumannii, while the breakpoint of Enterobacteriaceae spp. to AMC was used for both P. aeruginosa and A. baumannii. ${ }^{12}$

\section{Phenotypic Detection of MBLs and ESBLs}

The MBL phenotype was confirmed by EDTA inhibitory assay, and $\mathrm{a} \geq 5 \mathrm{~mm}$ increase in the zone diameter for meropenem vs the zone diameter for meropenem plus EDTA demonstrated MBL positivity. ${ }^{13}$ The phenotypic detection of ESBL production was detected following the procedures of the double-disk synergy test (DDST) ${ }^{14}$

\section{Resistance Genes Encoding MBLs and ESBLs}

Carbapenem-resistance genes, including $b l a_{\mathrm{NDM}}, b l a_{\mathrm{IMP}}$, $b l a_{\mathrm{VIM}}, b l a_{\mathrm{GIM}}, b l a_{\mathrm{SIM}}$ and $b l a_{\mathrm{SPM}}$, were screened by PCR and sequencing. ${ }^{15,16}$ The genotypes of the ESBL families, such as bla $a_{\mathrm{TEM}}, b l a_{\mathrm{SHV}}, b l a_{\mathrm{CTX}-\mathrm{M}-1}$ group, $b l a_{\text {СТХ-м-2 }}$ group, $b l a_{\text {СтХ-м-8 }}$ group, $b l a_{\text {СТХ-м-9 }}$ group, $b l a_{\mathrm{OXA}-10}$ and $b l a_{\mathrm{PER}}$, were confirmed by PCR and the sequencing primers listed in Supplemental Table 1.

\section{Checkerboard Microdilution Assay}

The checkerboard microdilution assay was used to determine the combined effect of ATM and AMC in vitro against isolates that produce MBL according to a previous study. ${ }^{17}$ The fractional inhibitory concentration index (FICI) was calculated, and the interpretation was proposed as follows: FICI $\leq 0.5$, synergy; $>0.5$ to $<1$, partial synergy; 1 , additive; $>1$ to $<4$, indifference; and $\geq 4$, antagonism.

\section{Time-Kill Assay}

Four isolates of MBL-producing Enterobacteriaceae spp., including three K. pneumoniae (Kpn1, Kpn6 and Kpn8) and one E. cloacae (Ec16), were selected for the time-kill assay. The bactericidal activity of the ATM and AMC combination in vitro was tested as previously reported with minor modifications. ${ }^{18}$ Briefly, bacteria were shaken in cationadjusted Mueller-Hinton broth for $2 \sim 4$ hours until the 
$\mathrm{OD}_{600 \mathrm{~nm}}$ reached $0.6 \sim 0.8$. Then, the cell density was adjusted to $0.5 \mathrm{McF}$ arland standard (approximately $10^{8} \mathrm{CFU} / \mathrm{mL}$ ) and then diluted 100 times with cation-adjusted Mueller Hinton broth until the initial inoculum density was theoretically approximately $5 \times 10^{6} \mathrm{CFU} / \mathrm{mL}$. The bacterial numbers $(\mathrm{CFU} / \mathrm{mL})$ were counted on Luria-Bertani agar plates at the $0,1,2,4,6$ and 24 hour time points after shaking. Each isolate was tested in four replicates, and each experiment included five tested groups with different antibiotic combinations, including the control group, $1 \times$ MIC ATM group, $1 \times$ MIC ATM plus $1 /$ $2 \times$ MIC AMC group, $1 \times$ MIC ATM plus $1 \times$ MIC AMC group, and $1 \times$ MIC ATM plus $2 \times$ MIC AMC group. When the colonies were below the limit of detection, a minimal value of nine was used.

\section{Results and Discussion}

MBLs are one of the most dominant mechanism of carbapenem resistance and can be produced by diverse species of Gram-negative organisms (GNOs), such as Enterobacteriaceae spp. (especially Klebsiella pneumoniae and Escherichia coli), Pseudomonas aeruginosa, and Acinetobacter baumannii. ${ }^{4,19-21}$ We acquired a total of 59 MBL-positive GNOs, including 33 Enterobacteriaceae spp. (15 K. pneumoniae, one $K$. oxytoca, one $K$. oxytoca, 14 Enterobacter cloacae, one Enterobacter hormaechei and one Citrobacter freundii), 22 P. aeruginosa and 4 A. baumannii isolates in this study (Supplemental Table 2 and Supplemental Figure 1), and we found that K. pneumoniae, E. cloacae and $P$. aeruginosa were the predominant hosts that harbour MBL genes; among these species, E. cloacae was not commonly reported. $^{22}$

To date, over ten types of MBLs have been reported, among which New Delhi metallo- $\beta$-lactamase (NDM), Verona integron-encoded metallo- $\beta$-lactamase (VIM) and Imipenemase (IMP) are the top three ones throughout the world. ${ }^{4,15,21,23,24}$ We first screened the MBL genes by PCR and observed that 45 isolates were positive for $\operatorname{bla}_{\mathrm{IMP}}(76.2 \%)$, 7 for $b l a_{\mathrm{VIM}}(11.9 \%)$ and 7 for $b l a_{\mathrm{NDM}}(11.9 \%)$ (Table 1). Further sequencing results confirmed that $b l a_{\mathrm{IMP}}$ have five different genotypes, namely, $b l a_{\mathrm{IMP}-4}, b l a_{\mathrm{IMP}-25}, b l a_{\mathrm{IMP}-26}$, $b l a_{\mathrm{IMP}-69}$ and $b l a_{\mathrm{IMP}-45}$, and bla $a_{\mathrm{VIM}}$ have two genotypes, namely, $b l a_{\mathrm{VIM}-36}$ and $\left.b l a_{\mathrm{VIM}-2}\right)$, while $b l a_{\mathrm{NDM}-1}$ was the only genotype of bla $a_{\mathrm{NDM}}$ (Supplemental Figure 1). bla $a_{\mathrm{IMP}-4}$ and $b l a_{\mathrm{IMP}-25}$ were the most dominant MBL genes in clinical isolates of Enterobacteriaceae spp. and $P$. aeruginosa, respectively.

ATM is thought to be an effective antimicrobial agent against MBL-GNOs, but it remains controversial to use ATM

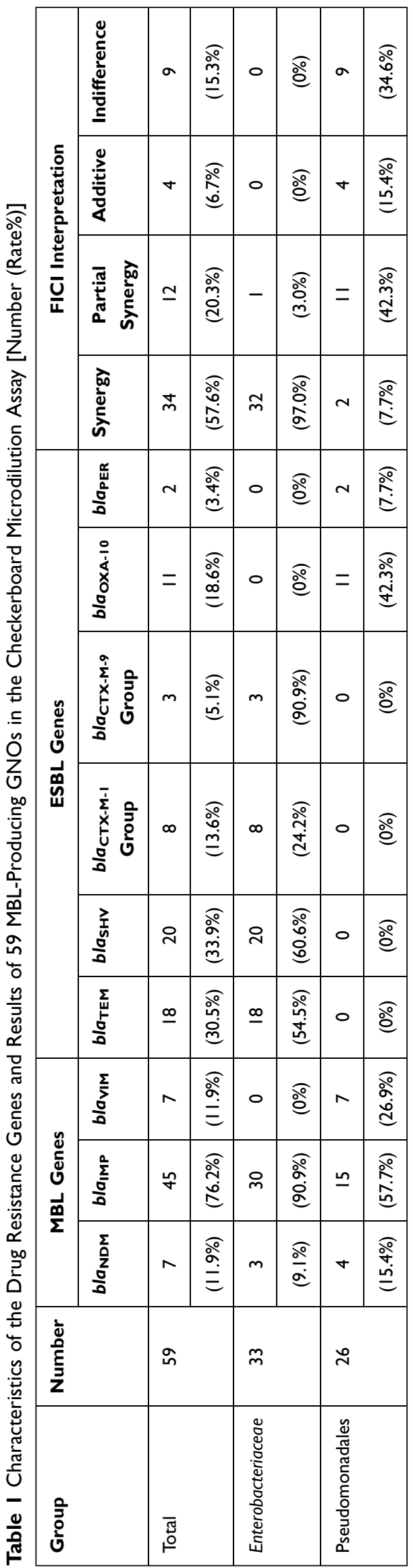




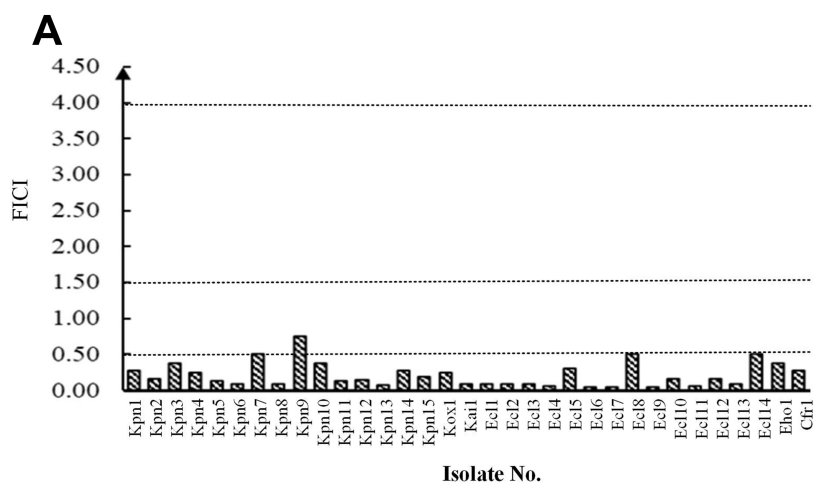

\section{C}

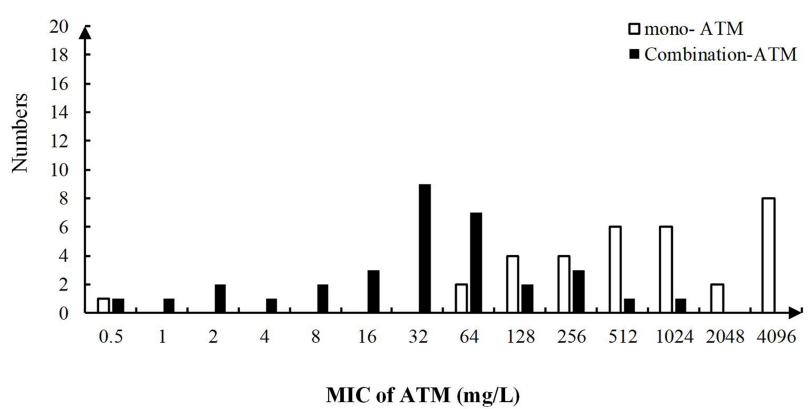

B

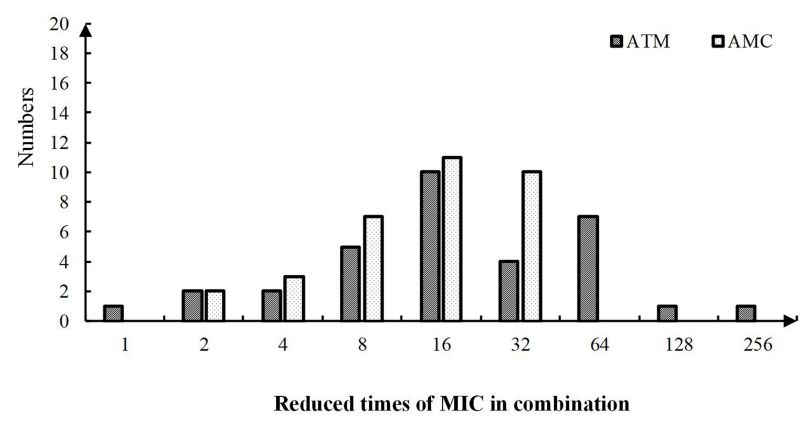

D

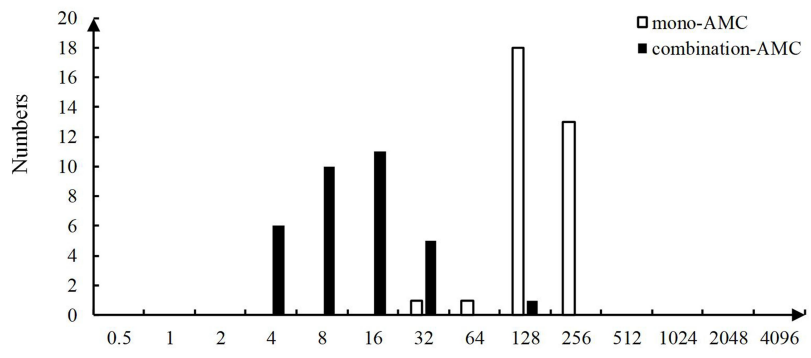

MIC of AMC (mg/L)

Figure I Results of the Enterobacteriaceae isolates in the checkerboard microdilution assay. (A) FICl values of Enterobacteriaceae isolates. The interpretation of the FICl was as follows: $\leq 0.5$, synergy; $>0.5$ to $<1$, partial synergy; =I, additive; $>$ I to $<4$, indifference. The dashed line is the line that divides the different interpretations. (B) Reduced times of the MIC $_{\text {ATM }}$ and MIC $_{\text {AMC }}$ in combination compared to the mono-antibiotic treatments. (C) MIC range of aztreonam in both the mono-ATM group and the combination group. (D) MIC range of amoxicillin/clavulanic acid both in the mono-AMC group and in the combination group.

alone because an increasing number of clinical isolates show resistance. ${ }^{25}$ All of our strains except for Kpn9 were resistant to ATM, with MICs ranging from $64 \mathrm{mg} / \mathrm{L}$ to $4096 \mathrm{mg} / \mathrm{L}$ for Enterobacteriaceae spp. isolates and from $8 \mathrm{mg} / \mathrm{L}$ to $256 \mathrm{mg} / \mathrm{L}$ for Pseudomonadales isolates (Supplemental Table 2). Similar to ATM, AMC is also ineffective in most cases, with MICs ranging from $32 \mathrm{mg} / \mathrm{L}$ to $256 \mathrm{mg} / \mathrm{L}$ for Enterobacteriaceae and from $64 \mathrm{mg} / \mathrm{L}$ to $2048 \mathrm{mg} / \mathrm{L}$ for Pseudomonadales (Supplemental Table 2). Our results, as well as those of other studies, suggested that most of the MBL-producing GNOs have developed potential mechanism to destroy ATM and render it useless when it was used as monotherapy. As a result, producing ESBLs was one of the most important mechanism. ${ }^{22}$

According to a recent report in China, the positive ratio of ESBL genes, including $b l a_{\mathrm{TEM}}, b l a_{\mathrm{SHV}}$, and $b l a_{\mathrm{CTX}-\mathrm{M}}$, was approximately $88.2 \%$ in CRE isolates. ${ }^{21}$ Taking the epidemic genotypes of ESBLs in our country into consideration, we screened six types of ESBL genes in Enterobacteriaceae isolates and Pseudomonadales isolates. There were four ESBL genotypes identified in 25 Enterobacteriaceae isolates with rates of $54.5 \%\left(b l a_{\mathrm{TEM}}\right), 60.6 \%\left(b l a_{\mathrm{SHV}}\right), 24.2 \%$ ( $b l a_{\mathrm{CTX}-\mathrm{M}-1}$ group) and $90.9 \%$ (bla CTX-M-9 $_{\text {g }}$ group), and 12 Pseudomonadales isolates harboured $b l a_{\mathrm{OXA}-10}$ or $b l a_{\mathrm{PER}}$ (Table 1, Supplemental Table 2).

AMC has been widely used in clinical practice and it is active against GNOs that produce ESBLs or AmpC $\beta$ lactamase due to the presence of clavulanic acid. ${ }^{26}$ Here, we combined AMC with ATM and used checkerboard microdilution assay to evaluate the effect of ATM and AMC in vitro with the fractional inhibitory concentration index (FICI). It was shown that the combination was synergistic against 32 Enterobacteriaceae isolates (97\%), with one exception against which the combination exhibited partial synergy (3\%) (Table 1, Figure 1A). Interestingly, the combination led to a significant MIC reduction in both ATM and AMC (Figure 1B). Compared to mono-antibiotic, the MICs of both ATM and AMC in combination generally decreased by 4 - to 64-fold and shifted entirely to the left lower MIC side (Figure 1B-D). The susceptibilities of ATM and AMC were restored in $4(12.5 \%)$ and $16(50 \%)$ isolates of the 32 Enterobacteriaceae isolates, respectively (Supplemental Table 2). Then, four isolates (Kpn1, Kpn6, Kpn8 and Ec16) were selected to determine the bactericidal effect of the combination, and a more rapid 

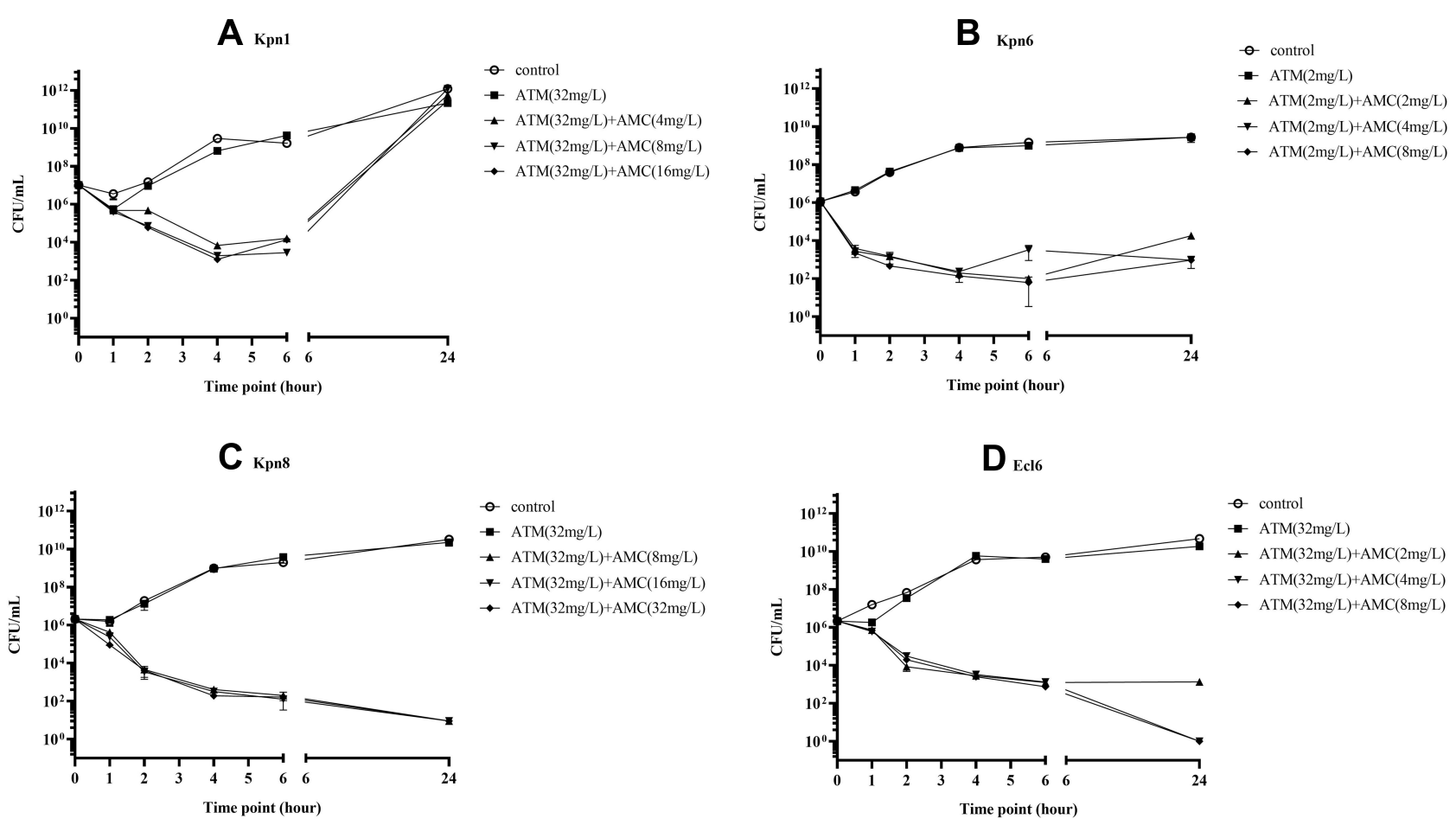

Figure 2 Time-kill curves of four representative Enterobacteriaceae isolates. (A) Isolate Kpn I; (B) isolate Kpn6; (C) isolate Kpn8; (D) isolate Ecl6. Each isolate was tested by five technical replicates and four biological replicates.

bactericidal effect was observed in the three combination groups than in the control group and mono-ATM group; this effect appeared in the first hour and led to a 5- to 7-fold reduction in $\log 10(\mathrm{CFU} / \mathrm{mL})$ at the 6 hour time-point in the time-kill assay (Figure 2). In summary, the results confirmed that the combination of ATM and AMC was efficient against Enterobacteriaceae isolates that coproduce MBL and ESBLs. ${ }^{811}$

P. aeruginosa and A. baumannii were the two species of Pseudomonadales isolates that harboured MBL genes in our research, and both of these species were thought to have complex and different mechanisms of resistance to AMC, such as $\beta$-lactamases and efflux pumps. ${ }^{27,28}$ In this study, $b l a_{\mathrm{OXA}-10}$ and/or $b l a_{\mathrm{PER}}$ were the main ESBL genes harboured by the $13 P$. aeruginosa isolates. The effects of the combined treatment of partial synergy and indifference were commonly observed against $P$. aeruginosa with ratios of $40.9 \%$ (9 isolates) and 36.4\% (8 isolates), respectively. In addition, among four A. baumannii isolates, the combined treatment caused a synergistic effect against only one isolate, a partial synergistic effect against two isolates and an indifference effect against one isolate (Supplemental Table 2, Figure 3A). Compared to that of mono-antibiotic against Pseudomonadales isolates, the MICs of the ATM and AMC combination presented lower values in most cases, with the MIC decreasing to over two dilution times (Figure 3B-D). The susceptibilities to ATM or AMC in combination were restored in 7 P. aeruginosa isolates (7/22, 38.2\%) and 3 A. baumannii isolates (3/4, 75\%) (Supplemental Table 2). Since the antimicrobial resistance due to multidrug efflux pumps was a major problem in Pseudomonadales isolates, the pump inhibitors might have a strong potential in application. ${ }^{29,30}$ In addition, the new molecules extracted from nature also showed power in antimicrobial activity including $P$. aeruginosa. ${ }^{31-35}$

In general, we provide evidence that the combination of ATM and AMC presents encouraging effects against clinical MBL-GNOs and might be a promising option to prevent the progression of drug resistance and to protect the use of ATM in the clinic. However, our results have some limitations that need to be considered. First, the conclusion of our research is based on an in vitro model, while the situation in vivo would be very different, since ATM and AMC have different PK/ PD parameters. Thus, more evidence to determine the appropriate ratio of ATM and AMC and more clinical experiences are still needed in the future. Second, AMC is weaker than ceftazidime/avibactam and meropenem/vaborbactam in terms of bactericidal effects both in vivo and in vitro. Therefore, patients who are suffering from severe infections, such as bloodstream infection, would obtain more benefits with those new agents. 


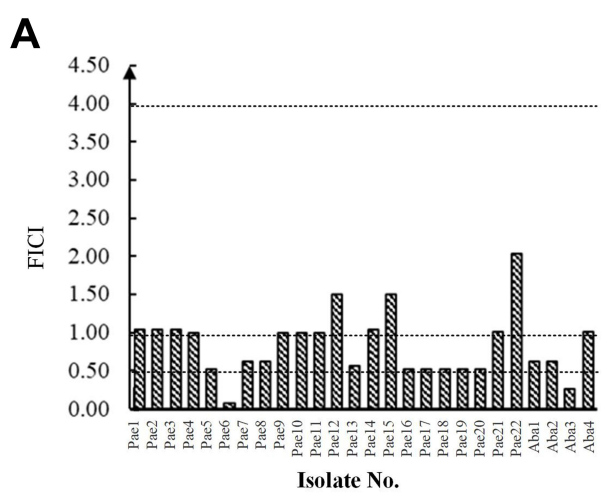

C

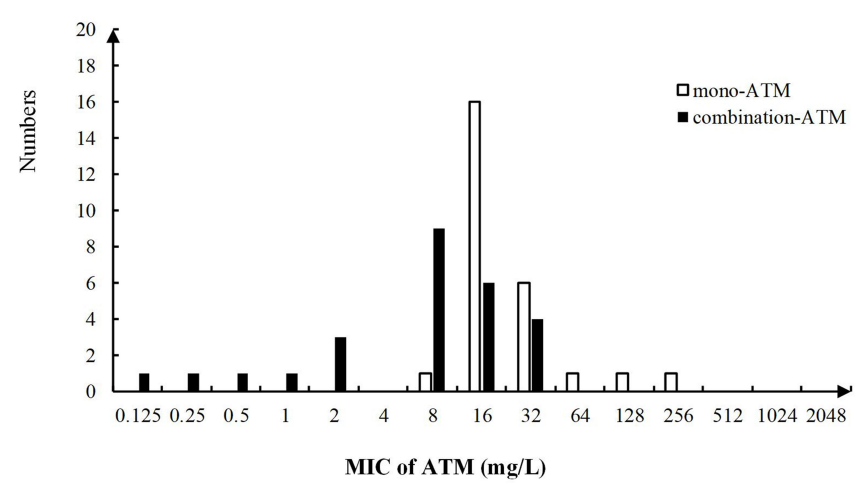

B

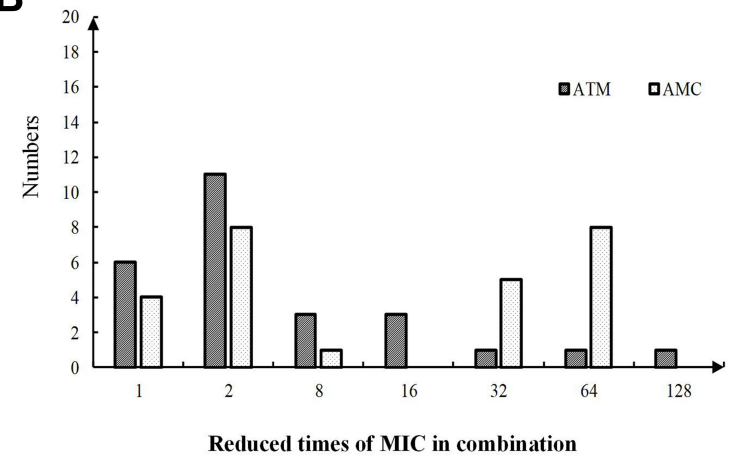

D

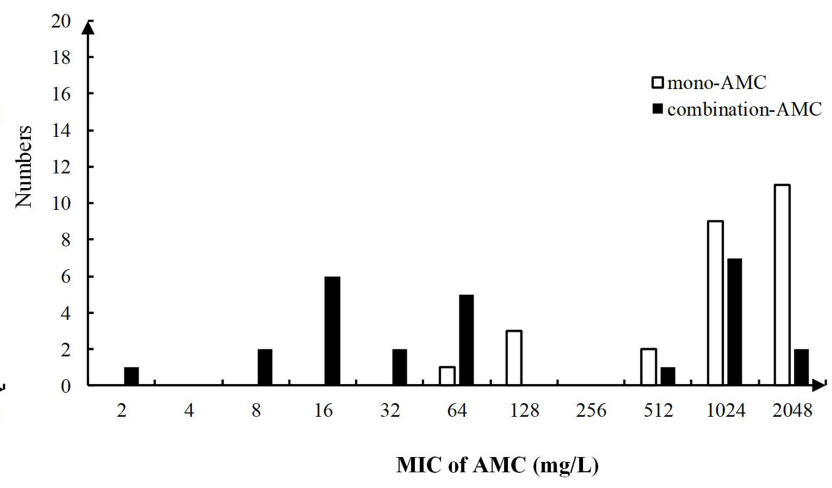

Figure 3 Results of the Pseudomonadales isolates in the checkerboard microdilution assay. (A) FICl values of Pseudomonadales isolates. The interpretation of the FICl was as follows: $\leq 0.5$, synergy; $>0.5$ to $<$ I, partial synergy; $=$ I, additive; $>$ I to $<4$, indifference. The dashed line is the line that divides the different interpretations. (B) Reduced times of the MIC $_{\text {ATM }}$ and MIC $_{\text {AMC }}$ in combination compared to the mono-antibiotic treatments. (C) MIC range of aztreonam in both the mono-ATM group and the combination group. (D) MIC range of amoxicillin/clavulanic acid both in the mono-AMC group and in the combination group.

\section{Conclusion}

Our study evaluated the combined effect of ATM and AMC against MBL-GNOs, and this combination demonstrated a dominant synergistic effect, especially against Enterobacteriaceae spp. isolates that coproduce ESBLs. This study strengthened the evidence for the use of ATM plus AMC against MBL-GNOs and provided important insights into the efficacy of clinical combination therapy.

\section{Ethics Approval}

The clinical isolates were part of the routine hospital laboratory procedure. The Ethics Committee of the Zhejiang Provincial People's Hospital exempted this study from review because the present study only focused on bacteria.

\section{Funding}

This work was supported by the National Natural Science Foundation of China (grant number 81601798), the National Natural Science Foundation of China (grant number 81772234) and the Zhejiang Provincial Natural Science Foundation (grant number LY20H200007).

\section{Disclosure}

The authors report no conflicts of interest in this work.

\section{References}

1. van Duin D, Doi Y. The global epidemiology of carbapenemase-producing Enterobacteriaceae. Virulence. 2017;8(4):460-469.

2. Logan LK, Weinstein RA. The epidemiology of carbapenem-resistant enterobacteriaceae: the impact and evolution of a global menace. J Infect Dis. 2017;215(suppl_1):S28-S36.

3. Karaiskos I, Lagou S, Pontikis K, Rapti V, Poulakou G. The "old" and the "new" antibiotics for MDR gram-negative pathogens: for whom, when, and how. Front Public Health. 2019;7:151.

4. Cornaglia G, Giamarellou H, Rossolini GM. Metallo-beta-lactamases: a last frontier for beta-lactams? Lancet Infect Dis. 2011;11(5):381-393.

5. Mojica MF, Papp-Wallace KM, Taracila MA, et al. Avibactam restores the susceptibility of clinical isolates of stenotrophomonas maltophilia to aztreonam. Antimicrob Agents Chemother. 2017;61(10).

6. Marshall S, Hujer AM, Rojas LJ, et al. Can ceftazidime-avibactam and aztreonam overcome beta-lactam resistance conferred by metallo-betalactamases in Enterobacteriaceae? Antimicrob Agents Chemother. 2017;61(4).

7. Biedenbach DJ, Kazmierczak K, Bouchillon SK, Sahm DF, Bradford PA. In vitro activity of aztreonam-avibactam against a global collection of gram-negative pathogens from 2012 and 2013. Antimicrob Agents Chemother. 2015;59(7):4239-4 248. 
8. Ract P. Synergistic in vitro activity between aztreonam and amoxicillinclavulanate against Enterobacteriaceae-producing class B and/or class $\mathrm{D}$ carbapenemases with or without extended-spectrum $\beta$-lactamases. J Med Microbiol. 2019;68(9):1292-1298. doi:10.1099/jmm.0.001052

9. Wenzler E, Deraedt MF, Harrington AT, Danizger LH. Synergistic activity of ceftazidime-avibactam and aztreonam against serine and metallo-beta-lactamase-producing gram-negative pathogens. Diagn Microbiol Infect Dis. 2017;88(4):352-354.

10. Livermore DM, Mushtaq S, Warner M, et al. Activities of NXL104 combinations with ceftazidime and aztreonam against carbapenemase-producing Enterobacteriaceae. Antimicrob Agents Chemother. 2011;55(1):390-394.

11. Emeraud C, Escaut L, Boucly A, et al. Aztreonam plus clavulanate, tazobactam, or avibactam for treatment of infections caused by metallo-beta-lactamase-producing gram-negative bacteria. Antimicrob Agents Chemother. 2019;63(5).

12. CLSI. Clinical and Laboratory Standards Institute. Performance Standards for Antimicrobial Susceptibility Testing. 29th ed. CLSI supplement M100. Wayne, PA: CLSI; 2020

13. Yong DE, Lee K, Yum JH, Shin HB, Rossolini GM, Chong YS. Imipenem-EDTA disk method for differentiation of metallo-betalactamase-producing clinical isolates of Pseudomonas spp. and Acinetobacter spp. J Clin Microbiol. 2002;40(10):3798-3801.

14. Jarlier V, Nicolas MH, Fournier G, Philippon A. Extended broad-spectrum beta-lactamases conferring transferable resistance to newer beta-lactam agents in Enterobacteriaceae: hospital prevalence and susceptibility patterns. Rev Infect Dis. 1988;10(4):867-878.

15. Fu Y, Du X, Ji J, Chen Y, Jiang Y, Yu Y. Epidemiological characteristics and genetic structure of blaNDM-1 in non-baumannii Acinetobacter spp. in China. J Antimicrob Chemother. 2012;67(9):2114-2122.

16. Ellington MJ, Kistler J, Livermore DM, Woodford N. Multiplex PCR for rapid detection of genes encoding acquired metallo-betalactamases. J Antimicrob Chemother. 2007;59(2):321-322.

17. Ji J, Du X, Chen Y, Fu Y, Wang H, Yu Y. In vitro activity of sulbactam in combination with imipenem, meropenem, panipenem or cefoperazone against clinical isolates of Acinetobacter baumannii. Int J Antimicrob Agents. 2013;41(4):400-401.

18. Keepers TR, Gomez M, Celeri C, Nichols WW, Krause KM. Bactericidal activity, absence of serum effect, and time-kill kinetics of ceftazidime-avibactam against beta-lactamase-producing Enterobacteriaceae and Pseudomonas aeruginosa. Antimicrob Agents Chemother. 2014;58(9):5297-5305.

19. Yan JJ, Ko WC, Chuang CL, Wu JJ. Metallo-beta-lactamaseproducing Enterobacteriaceae isolates in a university hospital in Taiwan: prevalence of IMP-8 in Enterobacter cloacae and first identification of VIM-2 in Citrobacter freundii. J Antimicrob Chemother. 2002;50(4):503-511.

20. Yu YS, Qu TT, Zhou JY, Wang J, Li HY, Walsh TR. Integrons containing the VIM-2 metallo-beta-lactamase gene among imipenem-resistant Pseudomonas aeruginosa strains from different Chinese hospitals. J Clin Microbiol. 2006;44(11):4242-4245.
21. Zhang B, Zhu Z, Jia W, et al. In vitro activity of aztreonam-avibactam against metallo-beta-lactamase-producing Enterobacteriaceae - a multicenter study in China. Int J Infect Dis. 2020;97:11-18.

22. Karlowsky JA, Kazmierczak KM, de Jonge BLM, Hackel MA, Sahm DF, Bradford PA. In vitro activity of aztreonam-avibactam against Enterobacteriaceae and pseudomonas aeruginosa isolated by clinical laboratories in 40 countries from 2012 to 2015. Antimicrob Agents Chemother. 2017;61:9.

23. Liang WJ, Liu HY, Duan GC, et al. Emergence and mechanism of carbapenem-resistant Escherichia coli in Henan, China, 2014. J Infect Public Health. 2018;11(3):347-351.

24. Cai Y, Chen C, Zhao M, et al. High prevalence of metallo-betalactamase-producing enterobacter cloacae from three tertiary hospitals in China. Front Microbiol. 2019;10:1610.

25. Ramsey C, MacGowan AP. A review of the pharmacokinetics and pharmacodynamics of aztreonam. J Antimicrob Chemother. 2016;71 (10):2704-2712.

26. Brown AG, Butterworth D, Cole M, et al. Naturally-occurring beta-lactamase inhibitors with antibacterial activity. $J$ Antibiot (Tokyo). 1976;29(6):668-669.

27. Pang Z, Raudonis R, Glick BR, Lin TJ, Cheng Z. Antibiotic resistance in Pseudomonas aeruginosa: mechanisms and alternative therapeutic strategies. Biotechnol Adv. 2019;37(1):177-192.

28. Lee CR, Lee JH, Park M, et al. Biology of acinetobacter baumannii: pathogenesis, antibiotic resistance mechanisms, and prospective treatment options. Front Cell Infect Microbiol. 2017;7:55.

29. Blanco P, Sanz-Garcia F, Hernando-Amado S, Martinez JL, AlcaldeRico M. The development of efflux pump inhibitors to treat gram-negative infections. Expert Opin Drug Discov. 2018;13 (10):919-931.

30. Usai D, Donadu M, Bua A, et al. Enhancement of antimicrobial activity of pump inhibitors associating drugs. J Infect Dev Ctries. 2019;13(2):162-164.

31. Bua A, Usai D, Donadu MG, et al. Antimicrobial activity of Austroeupatorium inulaefolium (H.B.K.) against intracellular and extracellular organisms. Nat Prod Res. 2018;32(23):2869-2871.

32. Donadu M, Usai D, Pinna A, et al. In vitro activity of hybrid lavender essential oils against multidrug resistant strains of Pseudomonas aeruginosa. J Infect Dev Ctries. 2018;12(1):9-14.

33. Amorese V, Donadu M, Usai D, et al. In vitro activity of essential oils against Pseudomonas aeruginosa isolated from infected hip implants. J Infect Dev Ctries. 2018;12(11):996-1001.

34. Le NT, Donadu MG, Ho DV, et al. Biological activities of essential oil extracted from leaves of Atalantia sessiflora Guillauminin Vietnam. J Infect Dev Ctries. 2020;14(9):1054-1064.

35. Donadu MG, Trong Le N, Viet Ho D, et al. Phytochemical compositions and biological activities of essential oils from the leaves, rhizomes and whole plant of Hornstedtia bella Skornick. Antibiotics (Basel). 2020;9(6)
Infection and Drug Resistance

\section{Publish your work in this journal}

Infection and Drug Resistance is an international, peer-reviewed openaccess journal that focuses on the optimal treatment of infection (bacterial, fungal and viral) and the development and institution of preventive strategies to minimize the development and spread of resistance. The journal is specifically concerned with the epidemiology of antibiotic resistance and the mechanisms of resistance development and diffusion in both hospitals and the community. The manuscript management system is completely online and includes a very quick and fair peerreview system, which is all easy to use. Visit http://www.dovepress.com/ testimonials.php to read real quotes from published authors. 